\title{
A condition of unknown significance: esophageal melanocytosis in a patient with celiac disease
}

A 74-year-old woman was admitted to our unit with complaints of abdominal discomfort and heartburn over 2 months. She had a history of hypertension and celiac disease, but admitted that she did not comply with a gluten-free diet. She had no history of any medication, smoking, or drinking alcohol, and her family history was unremarkable.

Physical examination findings were normal. Serological studies revealed positivity for anti-tissue transglutaminase, antigliadin, and anti-endomysial antibodies. An upper gastrointestinal endoscopy showed multiple, focal, flat and black pigmented areas in the middle and distal esophagus ( Fig.1 a). Multiple biopsies were obtained from these areas. In addition, atrophic folds and scalloping were observed in the second part of the duodenum during the endoscopic examination ( Fig.1b). Histological examination of esophageal biopsies showed melanin deposits in the basal layer and lamina propria of the squamous epithelium ( Fig.2a). Duodenal biopsy specimens revealed lymphocyte infiltration, crypt hyperplasia, and villous atrophy, compatible with celiac disease grade $\amalg b$ according to the Marsh classification ( $\bullet$ Fig. 2 b) $[1,2]$.

Esophageal melanocytosis is a benign and rare condition characterized by melanocytic proliferation in the basal layer of esophageal squamous epithelium, with an increased aggregation of melanin pigments. The etiology and pathogenesis of esophageal melanocytosis remain uncertain; however, it may be caused by gastroesophageal reflux or other conditions that lead to continued and chronic stimuli at the esophageal mucosa. It has also been reported to be associated with Addison's disease, Laugier-Hunziger syndrome, oral melanoma, esophageal squamous cell carcinoma, and celiac disease [3]. There are insufficient data to establish a guideline regarding treatment and surveillance of esophageal melanocytosis. However, the most important point is that esophageal melanocytosis must be differentiated from malignant melanoma [4].

Endoscopy_UCTN_Code_CCL_1AB_2AC

Competing interests: None

\section{Mevlut Hamamci, Fatih Karaahmet, Baris Yilmaz, Akif Altinbas, Sahin Coban}

Department of Gastroenterology, Dışkapı Yıldırım Beyazıt Educational and Research Hospital, Ankara, Turkey

\section{References}

1 Dewar DH, Ciclitira PJ. Clinical features and diagnosis of celiac disease. Gastroenterology 2005; 128: 19-24

2 Taavela J, Kurppa K, Collin P et al. Degree of damage to the small bowel and serum antibody titers correlate with clinical presentation of patients with celiac disease. Clin Gastroenterol Hepatol 2013; 11: 166 -171

3 Chang $F$, Deere $H$. Esophageal melanocytosis morphologic features and review of the literature. Arch Pathol Lab Med 2006; 130 : $552-557$

4 Yoshikane H, Suzuki T, Yoshioka N et al. Primary malignant melanoma of the esophagus presenting with massive hematemesis. Endoscopy 1995; 27: 397-399

\section{Bibliography}

Dol http://dx.doi.org/

10.1055/s-0034-1377546

Endoscopy 2014; 46: E469

(C) Georg Thieme Verlag KC

Stuttgart - New York

ISSN 0013-726X

\section{Corresponding author}

\section{Fatih Karaahmet, MD}

Department of Gastroenterology

Dışkapı Yıldııım Beyazıt Educational and Research Hospital

06080 Altındag

Ankara

Turkey

Fax: $+90-312-3186690$

fatih_ares@yahoo.com.tr

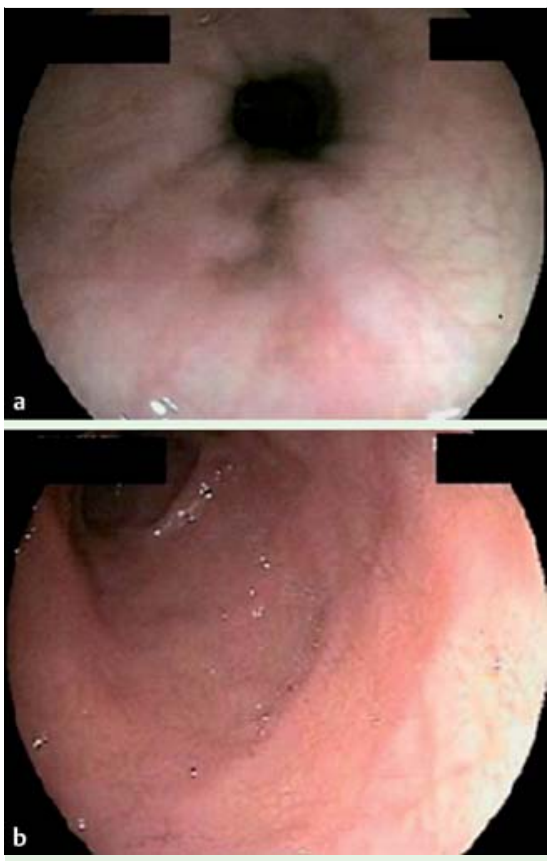

Fig. 1 Endoscopy images. a Multiple, focal, flat and black pigmented areas in the middle and distal esophagus. b Atrophic folds and scalloping in the second part of the duodenum.
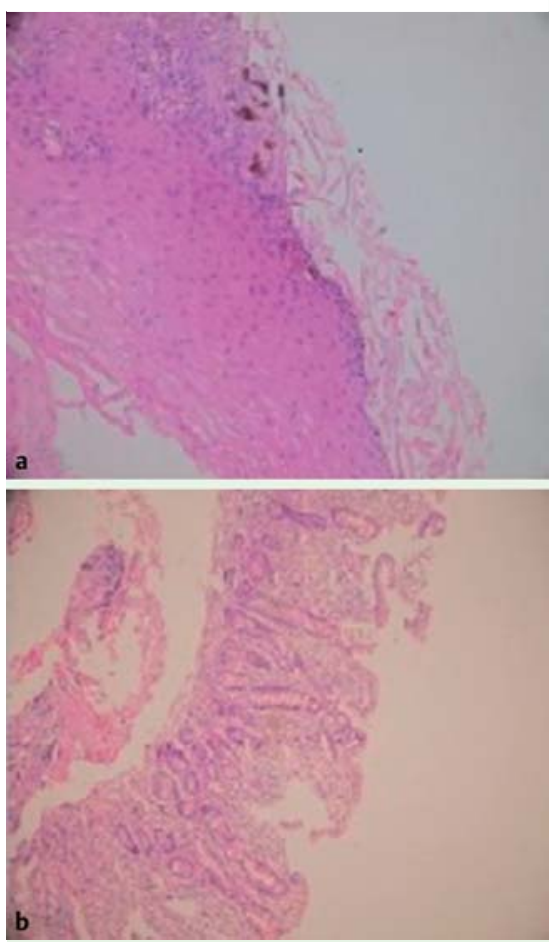

Fig. 2 Histological appearance of biopsies. a Esophagus - melanin deposits in the basal layer and lamina propria of the squamous epithelium. b Duodenum - lymphocyte infiltration, crypt hyperplasia, and villous atrophy in the mucosa, compatible with celiac disease. 